\section{Atenção à Saúde e Mortalidade \\ Neonatal: estudo caso-controle \\ realizado em Campinas, SP}

\section{Health care and neonatal mortality}

\section{Resumo}

Estudo caso-controle desenvolvido para identificar o efeito dos fatores associados à mortalidade neonatal, analisando variáveis socioeconômicas, de morbidade materna e, em especial, aquelas relacionadas à atenção à saúde. O estudo, realizado em Campinas, SP, incluiu 117 casos e 234 controles. As informações foram obtidas por meio de entrevistas domiciliares. A análise de regressão logística múltipla com modelo hierarquizado identificou como fatores associados ao óbito neonatal as variáveis de nível socioeconômico e de condições da família (renda, naturalidade e número de moradores do domicílio); as variáveis de morbidade materna (sangramento vaginal e parto antecipado por problema de saúde); e as variáveis de atenção à saúde (número de orientações recebidas durante o pré- natal, escolha do hospital do parto e tempo decorrido entre a internação e o parto) e de condições do recém-nascido (idade gestacional, peso ao nascer e Apgar no quinto minuto). O número de consultas de pré-natal não se manteve associado ao óbito neonatal após este ser controlado pela duração da gestação. Conclusão: O estudo aponta a necessidade de melhora da qualidade da atenção pré-natal, especialmente para as gestantes de pior condição socioeconômica e em risco de parto prematuro.

Palavras-chave: Mortalidade neonatal. Fatores de risco. Estudo de caso-controle.
Solange Duarte de Mattos Almeida

Secretaria Municipal de Saúde de Campinas

Avenida Anchieta, 200 - Campinas, SP

CEP 13015-904

somattos@uol.com.br
Marilisa Berti de Azevedo Barros

Departamento de Medicina Preventiva e Social

Faculdade de Ciências Médicas

Universidade Estadual de Campinas 
A Case control study was developed to identify the main factors associated with neonatal mortality, by analyzing socio-economic, maternal morbidity and especially health care related variables. Data were obtained from household interviews in Campinas, São Paulo, Brazil, 2001-2002. The study included 117 cases and 234 controls. The multiple logistic regression analysis in a hierarchic model identified the following as associated to neonatal death risk: socioeconomic variables (income, immigration, number of dwellers, no choice of delivery hospital); maternal morbidity (vaginal bleeding, early delivery due to health problems; time elapsed between hospital admission and delivery); quality of prenatal care (number of orientations received); health status of the newborn (gestational age, low birth weight and 5minute APGAR). Conclusion: This study indicates the need to improve prenatal care, mainly for pregnant women with poor socioeconomic status and those in risk of preterm delivery.

Key Words: Neonatal mortality. Risk factors. Case control study.

\section{Introdução}

O monitoramento da mortalidade neonatal, da mortalidade pós-neonatal e de seus fatores de riscos é essencial para identificar possíveis impactos de mudanças sociais e econômicas e dos avanços, e eventuais retrocessos, da cobertura e da qualidade dos serviços de saúde. O estudo dos fatores de risco dos óbitos infantis, compreendidos enquanto indicadores de várias dimensões das condições de vida, possibilita elucidar elementos da cadeia de eventos determinantes, identificar grupos expostos a diferentes constelações de fatores, e detectar diferenciadas necessidades de saúde em subgrupos populacionais, subsidiando as intervenções voltadas à redução dos óbitos neo e pós-neonatais ${ }^{1,2}$.

A mortalidade neonatal resulta de uma estreita e complexa relação entre as variáveis biológicas, sociais e de assistência à saúde, 0 que exige a proposição de modelos explicativos para a análise de seus determinantes.

A literatura científica da área maternoinfantil vem evidenciando, por meio de diferentes desenhos de estudo, uma série de fatores associados à mortalidade neonatal. Dentre os mais importantes e amplamente reconhecidos estão o peso ao nascer e a prematuridade $\mathrm{p}^{3-7}$. A redução do número de nascimentos com baixo peso é considerada uma estratégia eficaz para a prevenção da mortalidade neonatal ${ }^{8}$.

Outros fatores de risco para a morte neonatal encontrados na literatura, além das condições do recém-nascido (peso, prematuridade e Apgar), são as condições socioeconômicas e uma diversidade de características maternas como: raça/cor, idade, situação conjugal, antecedentes reprodutivos (paridade, intervalo interpartal, antecedentes de parto prematuro, de natimorto e de aborto e tipo de parto), morbidade (hipertensão, diabetes, infecção urinária, anemia, desnutrição, obesidade, entre outras) e o consumo de drogas, de bebidas alcoólicas e de tabaco ${ }^{8-14}$.

Também tem sido consistentemente evidenciada a associação entre o risco gestacional e indicadores do cuidado pré-natal, 
como o número de consultas realizadas, entre outros ${ }^{15,16}$. Entretanto, alguns autores têm assinalado a existência de vieses em algumas análises e questionado o efetivo papel do cuidado pré-natal nos resultados perinatais e neonatais observados e na prevenção do baixo peso ao nascer ${ }^{17,18}$.

Os níveis ainda elevados de mortalidade neonatal que ocorrem no Brasil apontam para a necessidade de melhor compreensão do papel da assistência no processo de determinação da saúde e da morbimortalidade neonatal. Serviços de saúde acessíveis e de boa qualidade deveriam ser capazes de reconhecer as mortes evitáveis e implementar medidas para reduzi-las.

O presente estudo tem como objetivo analisar os principais determinantes da mortalidade neonatal, em município com uma organizada rede básica de saúde e ampla disponibilidade de serviços terciários, buscando identificar, em especial, os fatores relacionados com a atenção à saúde.

\section{Material e Métodos}

Trata-se de um estudo caso-controle desenvolvido para identificar fatores associados ao óbito neonatal. O estudo foi realizado no município de Campinas (SP), que está localizado na região sudeste do Brasil e conta com uma população de 968.100 habitantes $^{19}$ (IBGE, 2000).

Foram considerados como "casos" todos os nascidos vivos que morreram antes de completar 28 dias de vida (mortes neonatais) no período compreendido entre $1^{\circ}$ de março de 2001 a 28 de fevereiro de 2002, cujo nascimento ocorreu em Campinas e eram filhos de mães residentes neste município. A relação dos óbitos neonatais ocorridos foi obtida do Banco de Dados sobre Mortalidade (SIM) da Secretaria Municipal de Saúde. Os "controles" foram selecionados por meio de sorteio aleatório (2 controles para cada caso) entre as crianças nascidas na mesma data que o caso e que sobreviveram ao vigésimo oitavo dia de vida. As listagens para sorteio foram obtidas do Banco de Dados de Nascidos Vivos (SINASC) da Secretaria Municipal de Saúde. Os controles também eram crianças nascidas em Campinas e filhos de mães residentes neste município.

O tamanho da amostra foi calculado para permitir detectar uma razão de odds de 2,5, considerando um poder do estudo (1- ß) de $80 \%$, um erro alfa de $5 \%$ e uma freqüência relativa de $10 \%$ de um dado fator de exposição. Considerou-se esse valor por tratar-se de um estudo em que vários fatores de exposição seriam analisados e as freqüências de alguns deles na população de origem eram desconhecidas. O cálculo do tamanho da amostra foi baseado em Schlesselman e Stolley ${ }^{20}$. O tamanho mínimo de amostra estimado foi de 108 casos e 216 controles aos quais acrescentou-se $10 \%$ para compensar possíveis perdas.

Para obtenção das informações foram realizadas entrevistas domiciliares com a mãe ou com o responsável pelo recém-nascido. As entrevistas foram realizadas entre junho de 2001 e maio de 2002, por três entrevistadores treinados. As variáveis analisadas neste estudo foram:

- condições socioeconômicas: renda, tipo de moradia, saneamento, número de cômodos, de quartos e de moradores, tempo de residência no domicílio, pai residindo no domicílio e ocupação e escolaridade da mãe;

- características demográficas e de morbidade maternas: idade, raça/cor, naturalidade, número de cidades em que morou, situação conjugal, problemas de saúde durante a gestação e internações;

- atenção pré-natal: local, semana gestacional de início do pré-natal, número de visitas, exames laboratoriais, ecografia, orientações recebidas durante as consultas ou em atividades em grupos (sobre ganho de peso, uso de medicamentos, evolução da gestação, sinais de parto, amamentação e vacinação), procedimentos clínicos realizados em todas as consultas (medida do peso, pressão arterial e altura uterina, ausculta dos batimentos cardíacos fetais, etc...);

- $\quad$ atenção ao parto: local, tipo de parto, pro- 
fissional que realizou o parto, intercorrências durante o parto e o tempo entre a admissão e o parto;

- condições do recém-nascido: sexo, peso, estatura, Apgar no $1^{\circ}$ e $5^{\circ}$ minutos e idade gestacional.

Os dados foram digitados em um banco de dados elaborado com o programa EpiInfo versão $6.02^{21}$. Na análise univariada foram estimados os valores de razões de odds, com intervalo de confiança de $95 \%$ com o uso do programa Epi-Info versão 6.02 ${ }^{21}$. Foi feita análise de regressão logística múltipla segundo modelo hierarquizado, usando o programa SAS System for Windows, versão 8.2, 1999-2001. Análises hierarquizadas têm sido propostas e utilizadas em diferentes estudos $^{22}$. Na análise hierárquica considerouse, para ordenação da introdução das variáveis no modelo, o nível hierárquico de cada variável de exposição na cadeia de determinação do óbito neonatal. O modelo proposto discriminou os fatores de risco hierarquicamente superiores como exercendo sua ação através daqueles situados inferiormente, obedecendo ao esquema descrito na Figura 1.

\section{Resultados}

Dos 129 óbitos neonatais ocorridos no período do estudo, a entrevista pode ser realizada em 117 casos. Os motivos das perdas foram: mudança para outro município (3), endereço não localizado (7) e recusa da família (2). Para cada caso foram entrevistados dois controles totalizando 351 entrevistas.

A Tabela 1 inclui as variáveis socioeconômicas e de trabalho materno que na análise univariada mostraram associações estatisticamente significativas com o óbito neonatal. Apresentaram maior risco de óbito os recém-nascidos cujas mães tinham renda familiar inferior a 2 salários mínimos $(\mathrm{OR}=1,94)$, eram migrantes ( $\mathrm{OR}=1,68)$, residiam em áreas de invasão $(\mathrm{OR}=2,33)$, em barracos $(\mathrm{OR}=2,08)$, em domicílios com menor número de cômodos $(\mathrm{OR}=2,63)$, com saneamento inadequado $(\mathrm{OR}=1,72) \mathrm{e}$ com 1 a $3(\mathrm{OR}=3,79)$ ou 7 e mais moradores $(\mathrm{OR}=2,05)$. Com relação à ocupação, mães que trabalhavam como empregadas domésticas apresentaram risco maior que as ocupadas em outras atividades $(\mathrm{OR}=2,27)$.

\section{Inserção socioeconômica}

\section{Condições da família, domicílio e de trabalho materno}
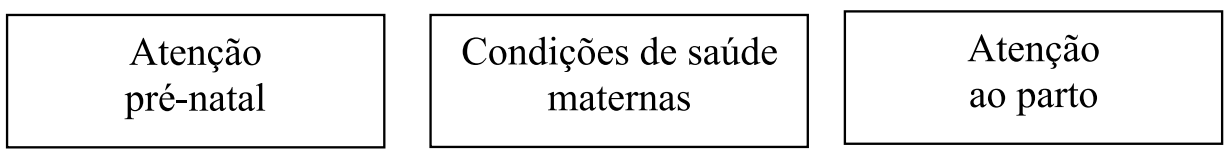

$$
\begin{aligned}
& \text { Condições de nascimento e } \\
& \text { saúde do recém nascido }
\end{aligned}
$$

Atenção ao recém-nascido

\section{Óbito neonatal}

Figura 1 - Modelo de análise do óbito neonatal

Figure 1 - Neonatal deaths analysis model 
Quanto ao período de trabalho, as mães que trabalharam apenas até 6 meses durante a gravidez $(\mathrm{OR}=5,11)$ e aquelas que não trabalharam $(\mathrm{OR}=2,10)$ tiveram riscos significativamente maiores que aquelas que trabalharam durante os nove meses de gestação.

A Tabela 2 apresenta as variáveis relativas à saúde materna que se mostraram significativas na análise univariada: presença de alguma doença durante a gestação $(\mathrm{OR}=2,00)$, tratamento de hipertensão arterial $(\mathrm{OR}=2,12)$, presença de sangramento vaginal $(\mathrm{OR}=3,23)$ e internações durante a gestação ( $\mathrm{OR}=2,29)$. A idade materna e as variáveis da história obstétrica (número de gestações, aborto, natimorto e filhos prematuros) não apresentaram significância estatística.

Quanto à atenção pré-natal (Tabela 3) apresentaram risco maior os recém-nascidos de mães que fizeram menos de 5 consultas $(\mathrm{OR}=7,15)$, que encontraram dificuldade para iniciar o pré-natal $(\mathrm{OR}=3,77)$, que não escolheram o médico $(\mathrm{OR}=1,71)$ e que não fizeram a maior parte das consultas com o mesmo médico $(\mathrm{OR}=1,82)$. Também apresentaram associações estatisticamente sig-

Tabela 1- Óbitos neonatais e controles, razão de odds e intervalos de confiança de 95\%, segundo variáveis socioeconômicas e de trabalho materno, Campinas, SP, 2001

Table 1 - Neonatal deaths and controls, odds ratio and 95\% confidence intervals, according to socioeconomic and maternal work variables

\begin{tabular}{|c|c|c|c|c|c|c|c|}
\hline \multirow[t]{2}{*}{ Variável } & \multicolumn{2}{|c|}{ Casos } & \multicolumn{2}{|c|}{ Controles } & \multirow[t]{2}{*}{$\mathrm{OR}$} & \multirow[t]{2}{*}{ IC $95 \%$} & \multirow[t]{2}{*}{$\mathrm{p}$} \\
\hline & $\mathrm{n}$ & $\%$ & $\mathrm{n}$ & $\%$ & & & \\
\hline \multicolumn{8}{|l|}{ Renda familiar (em salários mínimos) } \\
\hline $0-2$ & 32 & 27,4 & 38 & 16,2 & 1,94 & $1,13-3,31$ & 0,015 \\
\hline$>2$ & 85 & 72,6 & 196 & 83,8 & 1,00 & & \\
\hline \multicolumn{8}{|l|}{ Local de moradia } \\
\hline Áreas de Invasão/Ocupação & 20 & 17,1 & 19 & 8,1 & 2,33 & $1,19-4,56$ & 0,014 \\
\hline Outros & 97 & 82,9 & 215 & 91,9 & 1,00 & & \\
\hline \multicolumn{8}{|l|}{ Tipo de acabamento } \\
\hline Outros & 62 & 53,0 & 82 & 35,0 & 2,08 & $1,33-3,28$ & 0,001 \\
\hline Alvenaria completo & 55 & 47,0 & 152 & 65,0 & 1,00 & & \\
\hline \multicolumn{8}{|l|}{ Número de cômodos no domicilio } \\
\hline $1-2$ & 10 & 8,5 & 8 & 3,4 & 2,63 & $1,01-6,87$ & 0,047 \\
\hline 3 e mais & 107 & 91,5 & 226 & 96,6 & 1,00 & & \\
\hline \multicolumn{8}{|c|}{ Número de moradores no domicilio além do RN } \\
\hline $1-3$ & 66 & 56,4 & 66 & 28,2 & 3,79 & $2,25-6,41$ & $<0,001$ \\
\hline $4-6$ & 38 & 32,5 & 144 & 61,5 & 1,00 & & \\
\hline 7 e mais & 13 & 11,1 & 24 & 10,3 & 2,05 & $0,89-4,69$ & 0,061 \\
\hline \multicolumn{8}{|l|}{ Tipo de saneamento(água/lixo/esgoto) } \\
\hline Inadequado & 30 & 25,6 & 39 & 16,7 & 1,72 & $1,00-2,95$ & 0,048 \\
\hline Adequado & 87 & 74,4 & 195 & 83,3 & 1,00 & & \\
\hline \multicolumn{8}{|l|}{ Naturalidade da mãe } \\
\hline Migrante & 69 & 59,0 & 108 & 46,2 & 1,68 & $1,07-2,62$ & 0,024 \\
\hline Não migrante(Campinas e Região) & 48 & 41,0 & 126 & 53,8 & 1,00 & & \\
\hline \multicolumn{8}{|l|}{ Ocupação da mãe } \\
\hline empregada doméstica & 25 & 21,4 & 29 & 12,4 & 2,27 & $1,09-4,75$ & 0,017 \\
\hline outras ocupações & 30 & 25,6 & 79 & 33,8 & 1,00 & & \\
\hline Não trabalhou & 62 & 53,0 & 126 & 53,8 & 1,30 & $0,75-2,25$ & 0,327 \\
\hline \multicolumn{8}{|l|}{ Trabalho durante a gestação } \\
\hline até 6 meses & 37 & 31,6 & 31 & 13,2 & 5,11 & $2,40-10,97$ & $<0,001$ \\
\hline toda gestação & 18 & 15,4 & 77 & 32,9 & 1,00 & & \\
\hline Não trabalhou & 62 & 53,0 & 126 & 53,8 & 2,10 & $1,12-4,00$ & 0,013 \\
\hline
\end{tabular}


Tabela 2 - Óbitos neonatais e controles, razão de odds e intervalo de confiança de 95\%, segundo condições de saúde da mãe durante a gestação. Campinas, SP, 2001

Table 2 - Neonatal deaths and controls, odds ratio and $95 \%$ confidence intervals, according to health conditions of mother during pregnancy, Campinas, SP, 2001.

\begin{tabular}{|c|c|c|c|c|c|c|c|}
\hline \multirow[t]{2}{*}{ Variável } & \multicolumn{2}{|c|}{ Casos } & \multicolumn{2}{|c|}{ Controles } & \multirow[t]{2}{*}{ OR } & \multirow[t]{2}{*}{ IC $95 \%$} & \multirow[t]{2}{*}{$\mathrm{p}$} \\
\hline & $\mathrm{n}$ & $\%$ & $\mathrm{n}$ & $\%$ & & & \\
\hline \multicolumn{8}{|c|}{ Doença durante a gestação atual } \\
\hline Sim & 72 & 61,5 & 104 & 44,4 & 2,00 & $1,27-3,14$ & 0,003 \\
\hline Não & 45 & 38,5 & 130 & 55,6 & 1,00 & & \\
\hline \multicolumn{8}{|c|}{ Fez tratamento para pressão alta } \\
\hline Sim & 22 & 18,8 & 23 & 9,8 & 2,12 & $1,18-4,00$ & 0,020 \\
\hline Não & 95 & 81,2 & 211 & 90,2 & 1,00 & & \\
\hline \multicolumn{8}{|c|}{ Sangramento vaginal durante a gestação } \\
\hline Sim & 26 & 22,2 & 19 & 8,1 & 3,23 & $1,70-6,13$ & $<0.001$ \\
\hline Não & 91 & 77,8 & 215 & 91,9 & 1,00 & & \\
\hline \multicolumn{8}{|c|}{ Internação durante a gestação } \\
\hline Sim & 32 & 27,4 & 33 & 14,1 & 2,29 & $1,32-3,96$ & 0,003 \\
\hline Não & 85 & 72,6 & 201 & 85,9 & 1,00 & & \\
\hline
\end{tabular}

nificativas variáveis que são indicativas da qualidade da atenção pré-natal, tais como: número de orientações recebidas $(\mathrm{OR}=5,73$ para as que receberam até 2 orientações em comparação com as que receberam 6 ou 7 ) e realização dos procedimentos clínicos propostos $(\mathrm{OR}=7,99)$. As mães que não realizaram ecografia durante o pré-natal apresentaram um risco de 7,37 vezes o encontrado para as que a realizaram ao menos uma vez.

Quando é feita uma análise estratificada segundo a duração da gestação (Tabela 3 ), a associação entre o número de consultas de pré-natal e a mortalidade neonatal deixa de existir para os recém-nascidos a termo.

Com relação às variáveis de atenção ao parto (Tabela 4), tiveram maior risco os recém-nascidos de mães que apresentaram "sinais não usuais de parto" (hemorragias, edema, alterações de pressão) (OR=3,04), parto realizado em hospital não escolhido pela gestante $(\mathrm{OR}=5,04)$, realizado por médico plantonista $(\mathrm{OR}=1,76)$ e realizado em hospital escola (OR=2,10) Também apresentaram risco mais elevado os $\mathrm{RN}$ em que o parto ocorreu com menos de 1 hora ou com 10 ou mais horas após a internação $(\mathrm{OR}=2,66$ e $\mathrm{OR}=3,22$ respectivamente) em relação àquelas em que o parto ocorreu entre 1 e 10 horas a partir da internação.
Apresentaram maior risco de óbito neonatal (Tabela 5) os recém-nascidos de gestação múltipla $(\mathrm{OR}=15,88)$, com peso menor que 2.500 gramas ao nascimento $(\mathrm{OR}=24,37)$, com idade gestacional inferior a 37 semanas $(\mathrm{OR}=29,44)$ e Apgar no quinto minuto menor que 8 (OR=36,36).

Os resultados da análise de regressão logística múltipla não condicional hierarquizada estão apresentados na Tabela 6. Entre as variáveis socioeconômicas, permaneceu como estatisticamente significativa a renda familiar.

Das variáveis relacionadas às características da mãe, da família e do domicílio permaneceram significativas o número de pessoas no domicílio, o número de meses em que a mãe trabalhou durante a gestação e a condição de migração da mãe, quando ajustadas entre si e pela renda familiar.

Permaneceram estatisticamente significativas no bloco integrado por variáveis relacionadas à morbidade materna e à atenção ao pré-natal e ao parto as seguintes variáveis: número de visitas ao pré-natal, presença de sangramento vaginal, número de orientações recebidas na consulta de prénatal, parto antecipado por problema de saúde, escolha do hospital para o parto e o tempo entre a internação e o parto quando 
Tabela 3 - Óbitos neonatais e controles, razão de odds e intervalos de confiança de $95 \%$, segundo variáveis da atenção pré-natal, Campinas, SP, 2001

Table 3 - Neonatal deaths and controls, odds ratio and $95 \%$ confidence intervals, according to prenatal care variables, Campinas, SP, 2001.

\begin{tabular}{|c|c|c|c|c|c|c|c|}
\hline \multirow[t]{2}{*}{ Variável } & \multicolumn{2}{|c|}{ Casos } & \multicolumn{2}{|c|}{ Controles } & \multirow[t]{2}{*}{ OR } & \multirow[t]{2}{*}{ IC $95 \%$} & \multirow[t]{2}{*}{$\mathrm{p}$} \\
\hline & $\mathrm{n}$ & $\%$ & $\mathrm{n}$ & $\%$ & & & \\
\hline \multicolumn{8}{|l|}{ Número de visitas ao pré natal } \\
\hline 0 a 4 & 42 & 35,9 & 17 & 7,3 & 7,15 & $3,69-13,98$ & $<0,001$ \\
\hline 5 e mais & 75 & 64,1 & 217 & 92,7 & 1,00 & & \\
\hline \multicolumn{8}{|c|}{37 semanas ou mais (não prematuros) } \\
\hline 0 a 4 & 2 & 8,0 & 13 & 6,3 & 1,30 & $0,00-6,70$ & 0,736 \\
\hline 5 e mais & 23 & 92,0 & 195 & 93,7 & 1,00 & & \\
\hline \multicolumn{8}{|l|}{$<37$ semanas (prematuros) } \\
\hline 0 a 4 & 40 & 43,5 & 4 & 16,6 & 3,85 & $1,12-14,52$ & 0,015 \\
\hline 5 e mais & 52 & 56,5 & 22 & 83,4 & 1,00 & & \\
\hline \multicolumn{8}{|c|}{ Encontrou dificuldade para fazer o PN } \\
\hline Sim & 24 & 20,5 & 15 & 6,4 & 3,77 & $1,89-7,50$ & $<0,001$ \\
\hline Não & 93 & 79,5 & 219 & 93,6 & 1,00 & & \\
\hline \multicolumn{8}{|c|}{ Escolheu o médico que fez o PN } \\
\hline Não & 62 & 53,0 & 93 & 39,7 & 1,71 & $1,09-2,67$ & 0,019 \\
\hline Sim & 55 & 47,0 & 141 & 60,3 & 1,00 & & \\
\hline \multicolumn{8}{|c|}{ Consultas com o mesmo médico } \\
\hline Não & 36 & 30,8 & 46 & 19,7 & 1,82 & $1,09-3,01$ & 0,021 \\
\hline Sim & 81 & 69,2 & 188 & 80,3 & 1,00 & & \\
\hline \multicolumn{8}{|c|}{ Recebeu orientações durante o PN (número) } \\
\hline $0-2$ & 36 & 30,8 & 26 & 11,1 & 5,73 & $3,03-10,79$ & $<0,001$ \\
\hline $3-5$ & 43 & 36,8 & 72 & 30,8 & 2,19 & $1,29-3,67$ & 0,003 \\
\hline $6-7$ & 38 & 32,5 & 139 & 59,4 & 1,00 & & \\
\hline \multicolumn{8}{|c|}{ Realizou rotina clínica proposta no PN } \\
\hline Até 4 procedimentos & 11 & 9,4 & 3 & 1,3 & 7,99 & $2,18-26,21$ & 0,002 \\
\hline 5 e mais procedimentos & 106 & 90,6 & 231 & 98,7 & 1,00 & & \\
\hline \multicolumn{8}{|c|}{ Realizou exames de rotina (núm de exames) } \\
\hline até 7 & 15 & 12,8 & 16 & 6,8 & 2,00 & $0,95-4,21$ & 0,067 \\
\hline 8 e mais & 102 & 87,2 & 218 & 93,2 & 1,00 & & \\
\hline \multicolumn{8}{|c|}{ Realizou ecografia durante o PN } \\
\hline Não & 19 & 16,2 & 6 & 2,6 & 7,37 & $2,85-19,00$ & $<0,001$ \\
\hline Sim & 98 & 83,8 & 228 & 97,4 & 1,00 & & \\
\hline
\end{tabular}

ajustadas entre si e para as variáveis que persistiram nos blocos anteriores.

Com relação às características do recém nascido e suas condições de nascimento e saúde permaneceram estatisticamente significativas as variáveis: baixo peso ao nascer, prematuridade e Apgar no quinto minuto, ajustadas entre si e para as variáveis que persistiram dos blocos prévios. Nesta última etapa da análise hierárquica, quando foram incluídas as variáveis de condição do RN, permanecem significativas dos blocos anterio- res as variáveis: número de moradores no domicílio $\leq 3$; número de orientações recebidas durante o pré-natal $\leq 2$; parto precipitado por problema de saúde; escolha do hospital para o parto; e tempo entre a admissão no hospital e o parto $\leq 1$ hora.

\section{Discussão}

Neste estudo, o óbito neonatal foi analisado por meio de um desenho tipo casocontrole utilizando-se um modelo de análi- 
Tabela 4 - Óbitos neonatais e controles, razão de odds e intervalos de confiança de 95\%, segundo variáveis da atenção ao parto.Campinas,SP 2001

Table 4 - Neonatal deaths and controls, odds ratio and 95\% confidence intervals, according to care during delivery. Campinas, SP, 2001.

\begin{tabular}{|c|c|c|c|c|c|c|c|}
\hline \multirow[t]{2}{*}{ Variável } & \multicolumn{2}{|c|}{ Casos } & \multicolumn{2}{|c|}{ Controles } & \multirow[t]{2}{*}{ OR } & \multirow[t]{2}{*}{ IC $95 \%$} & \multirow[t]{2}{*}{$\mathrm{p}$} \\
\hline & $\mathrm{n}$ & $\%$ & $\mathrm{n}$ & $\%$ & & & \\
\hline \multicolumn{8}{|c|}{ Apresentou sinal antes da internação do parto } \\
\hline Sinais não usuais & 15 & 12,8 & 10 & 4,3 & 3,04 & $1,30-7,10$ & 0,010 \\
\hline Sinais usuais & 73 & 62,4 & 148 & 63,2 & 1,00 & & \\
\hline Não referiu nenhum sinal & 29 & 24,8 & 76 & 32,5 & 0,77 & $0,46-1,29$ & 0,325 \\
\hline \multicolumn{8}{|c|}{ Internação do parto precipitada por problema de saúde } \\
\hline Sim & 72 & 61,5 & 66 & 28,2 & 4,07 & $2,54-6,50$ & $<0,001$ \\
\hline Não & 45 & 38,5 & 168 & 71,8 & 1,00 & & \\
\hline \multicolumn{8}{|c|}{ Escolheu o hospital para realização do parto } \\
\hline Não & 44 & 37,6 & 25 & 10,7 & 5,04 & $2,88-8,80$ & $<0,001$ \\
\hline Sim & 73 & 62,4 & 209 & 89,3 & 1,00 & & \\
\hline \multicolumn{8}{|c|}{ Tempo decorrido entre a internação e o parto (em horas) } \\
\hline$<1$ & 35 & 29,9 & 41 & 17,5 & 2,66 & $1,53-4,59$ & 0,001 \\
\hline $1-9$ & 53 & 45,3 & 165 & 70,5 & 1,00 & & \\
\hline 10 e mais & 29 & 24,8 & 28 & 12,0 & 3,22 & $1,76-5,90$ & $<0,001$ \\
\hline \multicolumn{8}{|l|}{ Médico que realizou o parto } \\
\hline Médico plantonista & 93 & 79,5 & 161 & 68,8 & 1,76 & $1,03-2,97$ & 0,036 \\
\hline Médico do PN & 24 & 20,5 & 73 & 31,2 & 1,00 & & \\
\hline \multicolumn{8}{|l|}{ Estabelecimento do parto } \\
\hline Hospital-escola SUS & 43 & 36,8 & 52 & 22,2 & 2,10 & $1,16-3,80$ & 0,008 \\
\hline Hospitais privados SUS & 37 & 31,6 & 88 & 37,6 & 1,07 & $0,60-1,90$ & 0,810 \\
\hline Hospitais privados & 37 & 31,6 & 94 & 40,2 & 1,00 & & \\
\hline
\end{tabular}

Tabela 5 - Óbitos neonatais e controles, razão de odds e intervalos de confiança de 95\%, segundo condições de nascimento dos RNs.Campinas,SP, 2001

Table 5 - Neonatal deaths and controls, odds ratio and $95 \%$ confidence intervals, according to birth conditions of the newborn. Campinas, SP, 2001.

\begin{tabular}{|c|c|c|c|c|c|c|c|}
\hline \multirow[t]{2}{*}{ Variável } & \multicolumn{2}{|c|}{ Casos } & \multicolumn{2}{|c|}{ Controles } & \multirow[t]{2}{*}{ OR } & \multirow[t]{2}{*}{ IC 95\% } & \multirow[t]{2}{*}{$\mathrm{p}$} \\
\hline & $\mathrm{n}$ & $\%$ & $\mathrm{n}$ & $\%$ & & & \\
\hline \multicolumn{8}{|l|}{ Tipo de gestação } \\
\hline múltipla & 20 & 17,1 & 3 & 1,3 & 15,88 & $4,61-54,66$ & $<0,001$ \\
\hline única & 97 & 82,9 & 231 & 98,7 & 1,00 & & \\
\hline \multicolumn{8}{|c|}{ Idade gestacional no nascimento (em semanas) } \\
\hline$<37$ & 92 & 78,6 & 26 & 11,1 & 29,44 & $16,13-53,71$ & $<0,001$ \\
\hline 37 e mais & 25 & 21,4 & 208 & 88,9 & 1,00 & & \\
\hline \multicolumn{8}{|c|}{ Peso ao nascimento (em gramas) } \\
\hline até 2500 & 85 & 72,6 & 23 & 9,8 & 24,37 & $13,48-44,04$ & $<0,001$ \\
\hline mais de 2500 & 32 & 27,4 & 211 & 90,2 & 1,00 & & \\
\hline \multicolumn{8}{|c|}{ Apgar no $5^{\circ}$ minuto } \\
\hline $0-8$ & 81 & 69,2 & 15 & 6,4 & 36,36 & $18,60-71,06$ & $<0,001$ \\
\hline $9-10$ & 35 & 29,9 & 220 & 94,0 & 1,00 & & \\
\hline
\end{tabular}


Tabela 6 - Resultados da análise de regressão logística múltipla hierarquizada. Campinas, SP, 2001

Table 6 - Results of hierarchic multiple logistic regression analysis. Campinas, SP, 2001

\begin{tabular}{|c|c|c|c|c|}
\hline Modelo & OR bruta & OR ajustada & IC $95 \%$ & $\mathrm{p}$ \\
\hline \multicolumn{5}{|l|}{ Fatores socioeconômicos } \\
\hline \multicolumn{5}{|c|}{ Renda Familiar (em salários mínimos) } \\
\hline $0-2$ & 1,94 & 2,02 & $1,08-3,79$ & 0,027 \\
\hline$>2$ & 1,00 & 1,00 & & \\
\hline \multicolumn{5}{|c|}{ Características da mãe, da família e do domicilio * } \\
\hline \multicolumn{5}{|c|}{ Número de pessoas no domicílio } \\
\hline $1-3$ & 3,50 & 2,25 & $1,34-3,77$ & $<0,001$ \\
\hline 4 e mais & 1,00 & 1,00 & & \\
\hline \multicolumn{5}{|l|}{ Naturalidade da mãe } \\
\hline Outros Municípios & 1,90 & 2,39 & $1,29-4,44$ & 0,005 \\
\hline Campinas e região & 1,00 & 1,00 & & \\
\hline \multicolumn{5}{|c|}{ Trabalho durante a gestação\# } \\
\hline toda a gestação & 0,48 & 0,49 & $0,26-0,93$ & 0,005 \\
\hline até 6 meses & 2,43 & 3,38 & $2,05-5,58$ & 0,031 \\
\hline não trabalhou & 1,00 & 1,00 & & \\
\hline \multicolumn{5}{|c|}{ Condições de saúde da mãe, Atenção Pré natal e Atenção ao parto ** } \\
\hline \multicolumn{5}{|c|}{ Apresentou sangramento vaginal } \\
\hline Sim & 2,79 & 3,36 & $1,40-8,04$ & 0,006 \\
\hline Não & 1,00 & 1,00 & & \\
\hline \multicolumn{5}{|c|}{ Número de visitas ao $\mathrm{PN}^{\#}$} \\
\hline $0-4$ & 2,54 & 2,22 & $1,18-4,17$ & 0,013 \\
\hline 5 e mais & 1,00 & 1,00 & & \\
\hline \multicolumn{5}{|c|}{ Número de orientações recebidas na consulta de PN } \\
\hline 0 a 2 & 5,06 & 5,22 & $2,13-12,79$ & $<0,001$ \\
\hline 3 a 5 & 2,15 & 2,29 & $1,16-4,54$ & 0,017 \\
\hline 6 a 7 & 1,00 & 1,00 & & \\
\hline \multicolumn{5}{|c|}{ Internação para parto precipitada por problema } \\
\hline Sim & 4,59 & 4,94 & $2,64-9,24$ & $<0,001$ \\
\hline Não & 1,00 & 1,00 & & \\
\hline \multicolumn{5}{|c|}{ Escolheu o hospital para realização do parto } \\
\hline Não & 5,60 & 6,26 & $2,86-13,68$ & $<0,001$ \\
\hline Sim & 1,00 & 1,00 & & \\
\hline \multicolumn{5}{|c|}{ Tempo decorrido entre a internação e o parto (em horas) } \\
\hline$<1$ & 2,65 & 2,41 & $1,17-4,99$ & 0,017 \\
\hline $1-9$ & 1,00 & 1,00 & & \\
\hline
\end{tabular}

Condições de nascimento e de saúde do recém nascido ***

Idade gestacional ao nascimento (em semanas)

$<37$

37 e mais

Peso ao nascimento (em gramas)

até 2.500

2.500 e mais

Apgar no $5^{\circ}$ minuto

0 a 8

9 e 10
6,19

1,00

5,19

1,00

22,17

1,00
5,73

1,00

$1,83-17,98$

0,003

3,84

1,00

$1,18-12,50 \quad 0,025$

* OR ajustada pela variável renda familiar e pelas outras variáveis incluídas no modelo

** OR ajustada pelas variáveis do bloco anterior e pelas outras varáveis inclú́das no modelo

*** OR ajustada pelas variáveis do bloco anterior e pelas outras varáveis incluídas no modelo

\# estas variáveis são associadas à duração da gestação e seu efeito permanece apenas para os pré-termos se submetidas à análise estratificada (tabela3)

*OR adjusted to family income and to the other variables included in the model

**OR adjusted to the last block and to the other variables included in the model

***OR adjusted to the last block and to the other variables included in model

" these variables are associated with the length of gestation and the effect remains only for pre-term when submitted to stratified analysis (table 3) 
se hierarquizado para a identificação de fatores associados e de risco. Entre as variáveis socioeconômicas incluídas neste estudo mostraram-se associadas ao óbito neonatal a renda familiar (ajustada para escolaridade, bens e equipamentos eletrodomésticos, condições inadequadas de moradia e de saneamento básico), o número de moradores, a condição de migrante da mãe e o trabalho materno.

Resultados semelhantes a este estudo foram observados por Goldani e col. ${ }^{23}$ ao apontarem o excesso de mortalidade infantil (neonatal e pós neonatal) em populações de baixa renda familiar em Ribeirão Preto,SP. Leal e Szwarcwald ${ }^{24}$, utilizando um indicador sintético de condições socioeconômicas (nível educacional materno, renda familiar e proporção de domicílios com abastecimento adequado de água), observaram uma correlação direta e altamente significativa entre condições socioeconômicas e óbito neonatal no estado do Rio de Janeiro.

Diferentemente do esperado, filhos de mães residentes em domicílios com até 3 pessoas (excluindo o $\mathrm{RN}$ ) apresentaram um risco aumentado de morte neonatal quando comparados aos demais. Neste estudo, esta associação pode estar refletindo um conjunto de outros marcadores de baixa qualidade de vida. Os domicílios com até 3 moradores representaram $37,6 \%$ dos 351 incluídos no estudo, sendo que $14,0 \%$ tinham até 2 moradores e $0,9 \%$ até 1 . Comparando-se o perfil das famílias de domicílios com até 3 moradores com as demais, observou-se que as primeiras apresentaram menor tempo médio de residência (2,7 comparado a 6,7 anos; $(\mathrm{p}<0,001)$; maior percentual de mães com menos de 20 anos (28,8\% e 11,4\%; $\mathrm{p}=0,00007$ ); com renda familiar menor que 1 salário mínimo (15,9\% e 4,6\%; p=0,0006); com o chefe da família sendo a mãe $(12,9 \%$ e $4,1 \%$; $\mathrm{p}=0,005)$; mães solteiras $(22,7 \%$ e $18,7 \%$; $\mathrm{p}>0,05)$ e moradoras em "barracos" localizados em áreas de invasão $(6,8 \%$ e $4,6 \%$; $\mathrm{p}>0,05)$. São, portanto, famílias de mães mais jovens, residentes no domicílio há menos tempo, constituídas por mães sem companheiro, sem o suporte de uma família ampli- ada, que vivem em situação precária de habitação e com recursos insuficientes para a sua sobrevivência. Famílias muito pequenas, neste caso, estariam sinalizando expressões de carência e de exclusão social.

Dados da PNAD de 2001 (IBGE) mostram as diferenças significativas que vêm ocorrendo nos padrões de organização das famílias brasileiras, em especial em regiões metropolitanas do Sul e do Sudeste, onde se observa a redução do número médio de pessoas por domicílio (que passou de 4,5, em 1980, para 3,2 em 2001), o aumento do número de famílias chefiadas por mulheres sem cônjuge e com filhos, a diminuição do número de filhos e a permanência das desigualdades nos rendimentos.

A escolaridade da mãe tem sido apontada como um fator de risco importante para a mortalidade neonatal e para os nascimentos de baixo peso $0^{5,7,11,25,26}$. No presente estudo, não foi encontrada associação significativa entre a escolaridade materna e o óbito neonatal. Tampouco entre idade materna $\mathrm{e}$ morte neonatal. Resultados semelhantes foram observados no estudo de Morais Neto e Barros $^{2}$, no município de Goiânia, e no de Sarinho e col. ${ }^{27}$, em Recife.

A presença de morbidades maternas, como hipertensão arterial, diabetes, infecção do trato urinário e anemia, identificadas em outros estudos como fatores associados a pior resultado gestacional ${ }^{28,29}$, não mostrou associação com óbito neonatal depois de ajustadas para outras variáveis incluídas neste estudo. Somente a presença de sangramento vaginal em qualquer fase da gestação, internação para o parto precipitada por problema de saúde e o parto realizado até uma hora após a internação, permaneceram associados ao óbito neonatal. É possível que a presença de doenças e de hipertensão desencadeiem os eventos que permaneceram associados que seriam, desta forma, elementos intermediários no processo de determinação do óbito neonatal.

Com relação às variáveis relacionadas à atenção à saúde, observou-se neste estudo que apenas 7 gestantes $(1,9 \%)$ referiram não ter feito nenhuma visita ao pré-natal e todas 
eram mães de recém-nascidos que foram a óbito com menos de 28 dias de vida. A associação entre o número de consultas de prénatal e o resultado gestacional precisa considerar a duração da gestação. A forte associação entre visitas ao pré-natal e óbito neonatal, encontrada na análise univariada $(\mathrm{OR}=7,13)$, permanece significativa na análise estratificada apenas para os nascimentos com menos de 37 semanas de gestação (Tabela 3). Na análise de regressão logística, incluindo a idade gestacional, a associação entre consulta de pré-natal e óbito neonatal desaparece, indicando que a idade gestacional atua como fator de confundimento para a associação do óbito neonatal com o número de visitas ao pré-natal.

Inúmeras pesquisas vêm estudando o efeito do cuidado pré-natal na mortalidade perinatal, neonatal e infantil. Embora muitos questionamentos sejam feitos sobre o efeito benéfico do número de visitas ao pré-natal para a diminuição da morte no período perinatal e neonatal e para a prevenção dos nascimentos de baixo peso ${ }^{18,30}$, existe um razoável consenso na literatura científica em relação ao efeito negativo em termos de resultado gestacional associado à ausência de cuidado pré-natal e/ou um pré-natal realizado de forma inadequada ${ }^{17,31,32}$. Vintzileos e $\mathrm{col}^{33}$, analisando o impacto do cuidado prénatal nas mortes neonatais na presença e ausência de condições de alto risco materno, concluíram que o cuidado pré-natal está associado com a redução das mortes neonatais de forma mais acentuada para os nascimentos que ocorreram com 36 ou mais semanas de gestação e na presença de rotura prematura de membranas, placenta prévia, retardo de crescimento fetal e gravidez pós-termo, mesmo após ser ajustado para a idade gestacional, idade materna e peso ao nascer.

Poma $^{31}$, estudando o efeito do cuidado pré-natal nas taxas de mortalidade infantil em Chicago, também concluiu que a ausência de pré-natal, tanto quanto um cuidado inadequado, estiveram associados a um incremento na taxa de mortalidade infantil.

O número de consultas de pré-natal não garante a qualidade do cuidado, notadamente em locais onde a cobertura de pré-natal é alta. Nesta situação de alta cobertura é necessária a inclusão de outros critérios ou marcadores de qualidade, como foi preconizado pelo Ministério da Saúde, através do Programa de Humanização do Pré-natal e Nascimento (2000), que, além do mínimo de 6 consultas e início do pré-natal antes da $14^{\text {a }}$. semana de gestação, inclui a solicitação de todos os exames laboratoriais de rotina, realização de exame obstétrico, vacinação, exame citopatológico do colo uterino e orientações com relação à amamentação e ao parto.

A ausência ou o pequeno número de orientações recebidas durante $\mathrm{o}$ atendimento pré-natal, que permaneceu estatisticamente significativa no modelo final, é um indicador da qualidade da atenção pré-natal e está associado a outros critérios de adequação incluídos neste estudo, tais como: realização de exame clínico e obstétrico em todas as consultas, de todos os exames laboratoriais de rotina, de exame de ecografia e acompanhamento realizado sempre pelo mesmo médico, que na análise univariada mostraram-se fortemente associados com o óbito neonatal. Outros autores têm encontrado associação entre indicadores de qualidade do cuidado pré-natal e os resultados perinatais $^{31,33-35}$.

Com relação à opção de escolha para o hospital do parto, o município de Campinas oferece parto em 13 hospitais. Dois são hospitais-escola e outros dois são hospitais privados que atendem usuárias do SUS e de planos de saúde. O restante atende apenas usuárias de planos de saúde. Entretanto, apenas três destes hospitais concentram $70 \%$ dos partos, segundo dados da Secretaria Municipal de Saúde-Campinas. Para as gestantes que dependem do SUS, 30\% têm parto em hospital-escola. Das 70\% restantes, a maioria (92\%) utiliza a Maternidade, um hospital que tem uma localização geográfica privilegiada e goza de um ótimo conceito junto à população de gestantes. A possibilidade de escolha do hospital para o parto é restrita para as usuárias do SUS. Para as outras gestantes, a escolha deve ser bastante influenciada pelo médico que realiza o pré- 
natal e pelo próprio plano de saúde. Mas a maior parte das gestantes refere ter escolhido o hospital para o parto. Provavelmente porque aprovavam a indicação ou a opção oferecida. Como gestantes de melhor nível socioeconômico detêm maior grau de autonomia de escolha, a variável "escolha do hospital” seria um indicador de nível socioeconômico mais diretamente relacionado à atenção à saúde. Também é provável que pelo desenho do estudo, com informações obtidas retrospectivamente, a tendência de sentir que não pode escolher o local do parto seja maior para as gestantes cujos bebes vieram a falecer.

Com relação ao parto, não foi observada diferença de risco para morte neonatal entre os nascimentos ocorridos nos hospitais privados que atendem gestantes usuárias do SUS e os demais hospitais privados que atendem gestantes de planos de saúde. Resultado semelhante foi encontrado no estudo de Morais Neto e Barros² realizado em Goiânia. Para os nascidos em hospital-escola SUS, o risco de morte neonatal foi superior ao encontrado para os nascidos em hospitais privados. Este resultado pode ser explicado pelo fato de os hospitais-escola atenderem gestantes de maior risco e que apresentam condições socioeconômicas mais desfavoráveis.

As variáveis relativas às condições de nascimento e saúde do recém nascido que mostraram associação estatisticamente significativa foram: baixo peso, prematuridade e Apgar no quinto minuto $\leq 8$. Estas associações têm sido verificadas consistentemente na literatura ${ }^{25,17,26,27}$. O sexo do recém-nascido não mostrou associação com o óbito neonatal neste estudo, diferente do observado por Araujo e col. ${ }^{9}$ e por Menezes e col. ${ }^{17}$, que encontraram risco aumentado para os recém-nascidos do sexo masculino.

A porcentagem de baixo peso em Campinas vem se mantendo inalterada ao longo das ultimas décadas, com um crescimento proporcionalmente maior dos nascimentos prematuros $^{6}$. No presente estudo a porcen- tagem de baixo peso foi de $9,8 \%$ entre os controles e de $72,6 \%$ entre os óbitos neonatais. Do total de baixo peso $12,9 \%$ eram pequenos para a idade gestacional e $87,1 \%$ eram prematuros, mostrando a importância da diminuição do número de nascimentos prematuros para a redução da mortalidade neonatal.

É necessário ainda salientar os limites impostos pelo tipo de estudo utilizado, em que as informações foram obtidas após a ocorrência do evento morte. Mães que tiveram filhos que foram a óbito teriam maior tendência que as demais a avaliar negativamente os serviços recebidos durante o prénatal e o parto, e a valorizar mais intensamente os problemas de saúde ocorridos durante esse período.

Os resultados deste estudo apontam que recém-nascidos de gestantes de condições socioeconômicas desfavoráveis ainda apresentam maior risco de morte neonatal, nas condições de assistência à população estudada, e que a qualidade desta atenção revela-se associada à morte neonatal, mesmo após o ajuste para um conjunto expressivo de variáveis de distintas dimensões hierárquicas. O estudo reforça a importância do papel da qualidade da atenção oferecida pelos serviços de saúde durante o pré-natal e o parto, e a necessidade de implementar melhorias, em especial para as gestantes em piores condições socioeconômicas.

\section{Agradecimentos}

Ao Ministério da Saúde / FUNASA, pelo apoio no financiamento do trabalho de campo.

Aos dedicados entrevistadores: Washington, Neuza e Ana Rosa.

À Comissão de Estatística da FCM/ UNICAMP pela colaboração nas análises contidas neste trabalho.

A pesquisa foi aprovada pela Comissão de Ética da FCM-UNICAMP, processo número 186/2000. 


\section{Referências}

1. Cesar CLG. Fatores de Risco associados à mortalidade infantil em duas áreas da região metropolitana de São Paulo (Brasil) 1984-1985. Proposta de instrumentos preditivos. Rev Saúde Pública 1990; 24(4): 300-10.

2. Morais Neto OL, Barros MBA. Fatores de risco para mortalidade neonatal e pós neonatal na Região Centro-Oeste do Brasil: linkage entre bancos de dados de nascidos vivos e óbitos infantis. Cad Saúde Pública 2000; 16(2): 477-85.

3. Monteiro CA, Benicio MHA, Ortiz LPF. Tendência secular do peso ao nascer na cidade de São Paulo (1976 - 1998). Rev Saúde Publica 2000; 34(6): 26-40.

4. Victora CG, Smith PG, Vaughan JP, Nobre LC, Lombardi C, Teixeira AMB, et al. Influence of birth weight on mortality from infectious diseases: A case control study. Pediatrics 1998; 81(6): 807-11.

5. Almeida MF, Mello Jorge MHP. Pequenos para a idade gestacional: fator de risco para mortalidade neonatal. Rev Saúde Pública 1998; 32(3): 217-24.

6. Mariotoni GGB, Barros Filho AA. Peso ao nascer e características maternas ao longo de vinte e cinco anos na Maternidade de Campinas. J Pediatr 2000; 76 (1): 55-64.

7. Costa CE, Gotlieb SLD. Estudo Epidemiológico do peso ao nascer à partir da Declaração de Nascido vivo. Rev Saúde Pública 1998; 32(4): 328-34.

8. Ortiz LPF. Características da Mortalidade Neonatal no Estado de São Paulo. [tese de doutorado]. São Paulo. Faculdade de Saúde Pública da USP; 1999.

9. Araújo BF, Bozzetti MC, Tanaka ACA. Mortalidade neonatal precoce no município de Caxias do Sul. Um estudo de coorte. J Pediatr 2000; 76(3): 200-6.

10. Cunha AA, Reis AFF, Luz TP, Torres TZG. Complicações da gestação e do parto como fatores de risco de óbito perinatal. Rev Bras Ginecol Obstet 2000; 22(1): 19-26.

11. Haidar FH, Oliveira UF, Nascimento LF. Escolaridade materna : correlação com os indicadores obstétricos. Cad Saúde Pública 2001; 17(4): 1025-9.

12. Nucci LB, Duncan BB, Mengue SS, Brachetein LS, Schimidt MI, Fleck ET. Assessment of weight gain during pregnancy in general prenatal care services in Brazil. Cad Saúde Pública 2001; 17(6): 1367-74.

13. Lessa H, Bernardo G, Victora CG, Barros FC. Tabagismo em gestantes de área urbana da região sul do Brasil: 1982-1993. Rev Saúde Pública 1997; 31(3): 247-253.

14. Bottoms SF, Paul RH, Iams JD, Mercer BM, Thom EA, Roberts JM, et al. Obstetric determinants of neonatal survival: Influence of willingness toperform cesarean delivery on survival of extremely low-birth-weight infants. Am J Obstet Gynecol 1997; 176: 960-6.
15. Halpern R, Barros FC, Victora CG, Tomasi E. Atenção Pré-Natal em Pelotas, Rio Grande do Sul, Brasil,1993 / Prenatal care in Pelotas, Rio Grande do Sul, Brazil, 1993. Cad Saúde Pública 1998; 14(3): 487-92.

16. Trevisan MR, De Lorenzi DRS, Araújo NM, Esber K. Perfil da assistência pré-natal entre usuárias do Sistema Único de Saúde em Caxias do Sul. Rev Bras Ginecol Obstet 2002; 24(5): 293-9.

17. Menezes AMB, Barros FC, Victora CG, Tomasi E, Halpern R, Oliveira ALB. Fatores de risco para mortalidade perinatal em Pelotas, RS, 1993. Rev Saúde Pública 1998; 32(3): 209-16.

18. Lu MC, Tache V, Alexander GR, Kotelchuck M, Halfon N. Preventing low birth weight: is prenatal care the answer? J Matern Fetal Neonatal Med 2003; 13(6): 362 80 .

19. IBGE (Fundação Instituto Brasileiro de Geografia e Estatística), 2002. Censo Demográfico 2000. Rio de Janeiro: IBGE.

20. Schlesselman JJ. Case-control studies: design, conduct, analysis. Oxford University Press; 1982.

21. Dean AG et al. EPIINFO versão 6,04: A word processor, data base and statistics for epidemiology on micro computers. Atlanta, Georgia: Centers of Diseases Control, 1996.

22. Fuchs SC, Victora CG, Fachel J. Modelo hierarquizado: uma proposta de modelagem aplicada à investigação de fatores de risco para diarréia grave. Rev Saúde Pública 1996; 30(2): 168-78.

23. Goldani MZ, Barbieri MA, Bettiol H, Barbieri MR, Tomkins A. Infant mortality rates according to socioeconomic status in a Brazilian City. Rev Saúde Pública 2001; 35(3): 256-61.

24. Leal MC, Szwarcwald CL. Características da mortalidade neonatal no Estado do Rio de Janeiro na década de 80: uma visão espaço-temporal. Rev Saúde Pública 1997; 31(5): 457-65.

25. Grimmer I, Bührer C, Dudenhausen JW, Stroux A, Reiher $\mathrm{H}$, Halle $\mathrm{H}$ et al. Preconceptional factors associated with very low birthweight delivery in EAST and WEST Berlin: a case control study. BMC Public Health 2002; 2(1): 10.

26. Brito EMS. Estudo de caso controle em dois tempos para analisar os principais preditores de coeficiente de mortalidade neonatal intra-hospitalar das maternidades de Sorocaba,1995. [tese de doutorado]. São Paulo: Faculdade de Saúde Pública da USP; 2001.

27. Sarinho SW, Filho DAM, Silva GAP, Lima MC. Fatores de risco para óbitos neonatais em Recife: um estudo de caso-controle. J Pediatr 2001; 77(4): 294-8. 
28. Neves LAT. Fatores de risco para natimortalidade e neomortalidade precoce no município de Juiz de Fora. [tese de doutorado]. Rio de Janeiro: Instituto Fernandes Figueira; 2001.

29. Maranhão AGK, Joaquim MMC, Siu C, Kalume P, Castilho O, LEAL MC. Mortalidade perinatal e neonatal no Brasil. Tema Radis 1999; 17: 6-17.

30. Hueston WJ, Gilbert GE, Davis L, Sturgill V. Delayed prenatal care and the risk of low birth weight delivery. $J$ Community Health 2003; 28(3): 199-208.

31. Poma PA. Effect of prenatal care on infant mortality rates according to birth death certificate files. $J$ Natl Med Assoc 1999; 91(9): 515-20.
32. Kruger PM, Scholl TO. Adequacy of prenatal care and pregnancy outcome. J Am Osteopath Assoc 2000; 100(8): 485-92.

33. Vintzileos AM, Ananth CV, Smulian JC, Scorza WE, Knuppel RA. The impact of prenatal care on neonatal deaths in the presence and absence of antenatal highrisk conditions. Am J Obstet Gynecol 2002; 186(5): 1011-6.

34. Ribeiro VS, Moura da Silva AA. Tendências da mortalidade neonatal em São Luis, Maranhão, Brasil de 1979 a 1996. Cad Saúde Pública 2000; 16(2): 429-38.

35. Alexander GR, Kotelchuck M. Assessing the role and effectiveness of prenatal care: history, challenges, and directions for future research. Public Health Rep 2001; 116(4): 306-16.

recebido em: 05/11/2003

versão final reapresentada em: 29/01/2004

aprovado em: 04/02/2004 